\title{
The International Spinal Cord Injury Pediatric Activity and Participation Basic Data Set
}

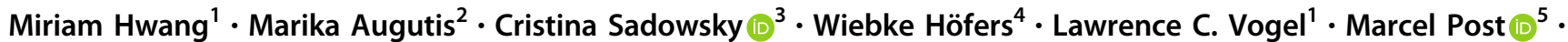

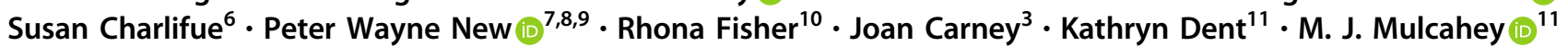

Received: 25 January 2019 / Revised: 6 August 2019 / Accepted: 11 August 2019

(c) The Author(s), under exclusive licence to International Spinal Cord Society 2019

\begin{abstract}
Study design International focus groups.

Objectives The objective of this project was to develop the International Spinal Cord Injury (SCI) Pediatric Activity and Participation (A\&P) Basic Data Set.

Methods A focus group of experts in pediatric and adult SCI, and contributors of the existing adult International SCI Adult A\&P Basic Data Set convened to develop an initial draft of the data set, which was iteratively refined over a 12 month period based on relevant literature and existing outcome measures that evaluate pediatric activity and participation. The draft was reviewed and approved by the larger project working group and then distributed to the International Spinal Cord Society (ISCoS), American Spinal Injury Association (ASIA), and relevant expert groups for review. Feedback received was considered before the final data set was approved.

Results The International SCI Pediatric A\&P Basic Data Set is comprised of 13 variables: administration date, mobility, dressing, feeding, toileting, communication, family outings, spending time with friends, being out with friends, participating in team or club activity, paid work, dating, and physical activity. It is intended for children between 6 and 17 years of age, who have been discharged from initial rehabilitation/hospitalization for a minimum of 3 months.

Conclusion The International SCI Pediatric A\&P Basic Data Set was developed to standardize the recording of a minimal amount of information about activities and participation in children with SCI. Further work on reliability and cultural validation is needed. Sponsorship This study was funded by the Rick Hansen Institute, Research Award \#G2015-27 (Mulcahey, PI).
\end{abstract}

\section{Introduction}

The International Spinal Cord Injury (SCI) Basic Data Sets were developed to collect the minimal amount of necessary

M. J. Mulcahey

Maryjane.mulcahey@jefferson.edu

1 Research Department, Shriners Hospitals for Children, Chicago, IL, USA

2 Department of Neurobiology, Core Sciences and Society, Karolinska Institute, Stockholm, Sweden

3 Rehabilitation Department, Kennedy Krieger Rehabilitation Institute, Baltimore, MD, USA

4 Physiotherapy Department, Sunnaas Hospital, Akershus, Norway

5 Center of Excellence for Rehabilitation Medicine, Brain Center Rudolf Magnus, University Utrecht and De Hoogstraat Rehabilitation, Utrecht, Netherlands information in areas relevant to an individual's medical concerns and psychosocial experience living with a SCI [1]. The Basic Data Sets have been developed on topics with high clinical relevance to the SCI community in order to
6 Research Department, Craig Hospital, Englewood, CO, USA

7 Spinal Rehabilitation Service, Caulfield Hospital, Melbourne, VIC, Australia

8 Epworth-Monash Rehabilitation Unit, Monash University, Melbourne, VIC, Australia

9 Department of Epidemiology and Preventitive Medicine, School of Public Health and Preventiative Medicine, Monash University, Melbourne, VIC, Australia

10 Fisher Association, Baltimore, MD, USA

11 Department of Occupational Therapy, Center for Outcomes and Measurement, Jefferson College of Rehabilitation Sciences, Jefferson (Philadelphia University+Thomas Jefferson University), Philadelphia, PA, USA 
promote standardized recording of clinical information, which can facilitate comparisons of injuries, treatments, and outcomes among individuals with SCI, treatment centers, and countries [2-4]. To date, one Core Data Set, which documents demographic and injury-related variables, and 20 basic data sets have been developed [2]. Efforts have been made to ensure that the international SCI basic data sets are applicable to pediatric age groups. This has been accomplished by having pediatric content experts serve on the working groups and via a formal review of each of the data sets for pediatric relevance [5].

The upper age limit defined for "pediatrics" ranges between 17 and 24, depending on source and context [6, 7]. An important consideration when conducting pediatric assessment and collection of clinical data, such as those collected by the international SCI basic data sets, is growth and development. Variables/responses that may be relevant to school-aged children and adolescent ages may have less relevance or not be relevant at all to preschool children. As an example, in the International SCI Basic Bowel Data Set Version 2.0 [8], the variable "awareness of need to defecate" is not appropriate for children who are not at the age at which continence is usually achieved (3-4 years old). On the other hand, some variables/responses may be relevant to each age across the pediatric ages, such as those in the spinal column and musculoskeletal basic data sets $[9,10]$. Growth and development must also be considered when deciding who (child, parent, and both) responds to questionnaires and interviews. For all of these reasons, one standard pediatric age cannot be defined for all variables across all data sets.

While many of the existing data sets can be used in pediatrics, following the review by Carroll et al., it was determined that several were not applicable to children of pediatric age groups [5]. Notably, the International Activity and Participation (A\&P) Basic Data Set [11] was considered inadequate for monitoring activity and participation in children 17 years of age and younger, and hence catalyzed the development of the data set described within this paper. The primary objective of the existing adult International SCI A\&P Basic Data Set Data Set [11] is to assess $\mathrm{A} \& \mathrm{P}$ in the following domains of the International Classification of Functioning, Disability, and Health (ICF) [12]: mobility; self-care; domestic life; social interactions and relationships; major life areas; community, social, and civic life. Development of a pediatricspecific activity and participation basic data set was recommended to better capture relevant and developmentally appropriate experiences of activity and participation of children [5]. The aim of this paper is to present the International SCI Pediatric A\&P Basic Data Set Version 1.0, discuss the process by which it was developed and the rationale for domains and variables.

\section{Methods}

An international interdisciplinary group of individuals with expertize in pediatric SCI care and research, and those who contributed to the development of the existing (adult) International Activity and Participation Basic Data Set was formed through invitation by the Principal Investigator (MJM). Work group members included individuals from Australia (1), the Netherlands (1), Norway (1), South Korea (1), Sweden (1), and the United States (5). The process used to develop the International Pediatric SCI A\&P Basic Data Set followed the iterative process used for developing all of the other International SCI Basic Data Sets [1]. Specifically:

1. Existing instruments for measurement of pediatric activity and participation [13-25] were reviewed from which an initial draft data set was developed. The initial draft contained three domains, activities of daily living (ADLs), physical activities, and participation, comprised of items adapted from The Spinal Cord Independence Measure Self-Report for Youth (SCIM-III SR-Y) [18], the Physical Activity Questionnaire for Older Children (PAQ-C) [16, 24], and the Pediatric Measure of Participation short forms [25]. Because an International SCI Education Basic Data Set [26] was being developed in parallel to the development of the International SCI Pediatric Activity and Participation, school participation variables were not included on the A\&P data set.

2. The group met face to face in a focus group format, and continued to review iterative drafts of the data set via biweekly conference calls and frequent email communications over a 1 year period (2017-2018).

3. A full draft was vetted during a follow up face-to-face meeting of the larger workgroup for development of Pediatric SCI/D Basic Data Sets, held in Philadelphia PA in February 2018, during which the larger pediatric work group reviewed and made recommendations to draft variables and their descriptions. Specifically, changes were made in content and wording of the variables to allow for ease of comprehension by both child and caregiver respondents, as well as to have greater applicability to the international community. The age criteria for several participation variables, specifically "go out with friends", "be on a team or club", "dating", and "paid work" was also discussed until there was consensus on age applicability for the majority of children living in regions across the globe.

4. The draft was sent to the American Spinal Injury Association (ASIA) Board and the International Spinal Cord Society (ISCoS) Executive and Scientific Committees, with a request to make the draft available 
on the respective websites for membership feedback. No additional suggestions or comments were made.

5. The Data Set was approved by ASIA Board and the ISCoS Executive and Scientific Committees.

\section{Results}

The final International SCI Pediatric A\&P Basic Data Set contains 13 variables corresponding to one of three domains: ADLs, participation, and physical activities. It was developed for children between 6 and 17 years of age, with traumatic or nontraumatic acquired SCI and who have been discharged from initial hospitalization/rehabilitation for at least 3 months. The 3 month period is a criteria for the PMoP [25] so that children have opportunities to perform activities to better aide in self-report. The data set can be administered through an interview, or by self-report (completing it via paper/pencil without an interviewer).

Based on previous cognitive interviewing studies including two that were conducted on the SCIM-III SR-Y [18] and PMoP [27], it is expected that children 8 years of age and older will be able to self-report and respond to variables on the International SCI Pediatric A\&P Data Set independently, and for children younger than 8 , a parent or caregiver proxy will respond on their child's behalf. As seen in clinical practice, parent or caregiver report may be used in combination with child report (in children 8-12 years of age) or be supplemental to child report (children 13-14 years of age). Parent input is optional when administrating the data set to children aged 15-17, assuming cognition and development are at age levels. At aged 18 the existing adult
International SCI A\&P Basic Data Set should be used. Together, these two data sets provide the opportunity to monitor activity and participation using standardized methods over the course of the child's lifetime.

As shown in Table 1, the International SCI Pediatric A\&P Basic Data Set contain domains that are similar to the domains on the adult A\&P Basic Data Set, however the variables, variable descriptors, and/or metrics differ. For instance, the ADL variables in both data sets record information about level of independence in mobility, dressing, feeding, and toileting activities, however the description for the pediatric variables and coding metrics are adopted from the SCIM-III SR-Y [18]. The ADL variables in the International SCI Pediatric A\&P Basic Set can be classified within the Mobility (d4) and Self-care (d5) domains of the International Classification of Disability for Children and Youth (ICF-CY) [28].

The Participation domain records areas important to community participation and psychosocial development in children and assesses the following domains of the ICFCYF [28]: communication (d3), interpersonal interactions and relationships (d7), major life areas (d8), and community, social, and civic life (d9) [29-31]. The physical activities domain considers the significant implications of participating in physical activity for both cardiovascular fitness and psychosocial health and wellness in children with disabilities [32-35]. The single physical activities variable was adapted from the PAQ-C $[16,24]$ and, can be defined by the Recreation and leisure category (d930) within the Community, social, and civic life domain (d9) of ICF-CY [28].

Each of the variables on the International SCI Pediatric A\&P Basic Data Set are described below.

Table 1 Comparison of items and performance metrics in the existing and Pediatric Activities and Participation International SCI Basic Data Sets

The International SCI Activities and Participation Basic Data Set

\begin{tabular}{|c|c|c|c|}
\hline Item & Performance metric & Item & Performance metric \\
\hline Mobility & Task execution & Mobility & Task execution \\
\hline Dressing & Task execution & Lower body dressing & Task execution \\
\hline Feeding & Task execution & Feeding & Task execution \\
\hline Toileting & Task execution & Toileting & Task execution \\
\hline Household Work & Hours a week & Physical activities & Times per week \\
\hline Volunteer Work & Hours a week & Communication & Frequency compared with peers \\
\hline Family Relationships & Number of contacts & Family outings & Frequency compared with peers \\
\hline Friends Relationships & Number of contacts & Time spent with friends & Frequency compared with peers \\
\hline Education & Hours a week & Outings with friends & Frequency compared with peers \\
\hline Spare time activities & Hours a week & Team activities & Frequency compared with peers \\
\hline Spousal relationships & Presence and type of relationship & Dating & Presence \\
\hline Paid work & Hours a week & Paid work & Frequency compared with peers \\
\hline
\end{tabular}

The International SCI Pediatric Activities and Participation Basic Data Set 


\section{Date of data collection}

The International Pediatric SCI Basic Data Set can be administered any time after 3 months post-discharge from initial rehabilitation/hospitalization. Three months was selected as this amount of time is considered minimal for children to have an opportunity to engage in activities to aide in self-report.

\section{Activities of daily living}

A 4-week recall is used for the ADL variables; the 4-week recall is also used for the existing adult International SCI A\&P Basic Data set. The ADL variables were obtained from the SCIM-III SR-Y [18]. The SCIM-III SR-Y was developed based on cognitive interview studies on the widely-implemented SCIM-III [36, 37] to evaluate level of self-reported independence by children with SCI. Only the four SCIM-III SR-Y items that correspond to the variables on the existing adult International SCI A\&P Basic Data Set were included in the pediatric A\&P data set This was done to provide a mechanism for monitoring ADL performance across the pediatric-adult lifespan.

\section{Mobility}

This variable records the mode of mobility used to move medium distances $(10-100 \mathrm{~m})$ in the last 4 weeks. Either "I use a wheelchair to move around" or "I walk medium distances" is selected. Walking can only be selected if the child can walk medium distances. Medium distances correlates to the distance between classrooms at school, length of a gymnasium, or a city block. Once the mode of mobility is established, the variable records the level of assistance that is required to move medium distances.

\section{Dressing}

This variable records level of independence in dressing/ undressing the lower body (clothes, shoes, and braces) in the last 4 weeks.

\section{Feeding}

This variable records level of independence in feeding (eating and drinking) in the last 4 weeks. Feeding (eating and drinking) is defined as cutting food, opening containers/ bags, pouring, bringing food to mouth, or holding a cup with fluid. The code "I eat nothing with my mouth" is used to record feeding via a gastrostomy or other type of parenteral feeding.

\section{Toileting}

This variable records the level of independence in toileting in the last 4 weeks. Toileting can occur either on the toilet or in bed and involves personal hygiene, adjusting clothes, using napkins, or diapers.

\section{Participation}

A 4 week recall is also used for the seven participation variables. The participation variables are drawn from the PMoP [25], which is a SCI-specific pediatric patient reported outcome instrument developed using item response theory $[38,39]$. While the PMoP evaluates participation based both on what the child desires and as compared with friends' participation, the International SCI Pediatric A\&P Basic Data Set only uses the comparison with friends. With the exception of the dating, each of the participation variables are coded as follows:

(1) I don't do it.

(2) I do it a lot less than my friends.

(3) I do it a little less than my friends.

(4) I do it as much as my friends.

\section{Communication}

This variable records the child's frequency (compared with friends) to which the internet and electronic devices are used to communicate with others. Electronic devices include, but are not limited to, computers, tablets, smartphones, game consoles, etc. Internet use via an electronic device for communication may include texting, email, messaging applications (such as Snapchat, WhatsApp, Messenger, etc.), and social media (such as Twitter, Facebook, Instagram, etc.). This variable may be coded not applicable (N/A) if the child does not have access to the internet or an internet-accessible electronic device.

\section{Family outings}

This variable records the frequency (compared with friends) to which the child goes places with family. Places may include restaurants, theater, sports events, holiday travel, etc.

\section{Spending time with friends}

This variable records the frequency (compared with friends) to which the child plays or hangs out at a friend's house. 


\section{Being out with friends}

This variable records the frequency (compared with friends) to which the child goes out with friends to eat, watch movies, attend concerts, or sports events. If the child is too young to be out without a parent or guardian the variable may be coded as N/A.

\section{Participating in team or club activity}

This variable records the frequency (compared with friends) to which the child participates on a community team or club. Community team or club activities include any sports leagues, girl/boy scouts, choir, theater, etc. This variable may be coded N/A if the child is less than 6 years of age.

\section{Dating}

This variable documents whether or not the child with SCI dates and/or has a girlfriend/boyfriend. The code "I do not date" means the individual does not go on dates or does not have a boyfriend/girlfriend, whereas the code "I date" means the individual dates or has a girlfriend or boyfriend. This variable may be coded as N/A if the child is too young to date or in cultures where dating is not usual (for example, arranged marriages).

\section{Paid work}

This variable records the frequency (compared with friends) to which the child engages in gainful employment or work. The preamble to this question (A job means doing something to get paid) highlights the requirement of "gainful" or "paid" employment/work. Examples of paid work can include activities such as babysitting, dog-walking, newspaper delivery, etc. Allowances for household chores are not considered paid work. If the child is too young to perform paid work or in cultures where paid employment is not usual, this can be coded as N/A.

\section{Physical activities}

This variable records the level of physical activity in the last 7 days. Things that involve little physical effort include sedentary activities such as reading, watching television, playing games, etc. This variable was adapted from the PAQ-C [16, 24], The 7-day recall period is unique to this variable; it is the same recall period used in the PAQ-C as well as other validated physical activity measures noted in the literature, including the Leisure Time Physical Activity Questionnaire for People with Spinal Cord Injury and the International Physical Activity Questionnaire [33, 40, 41].

\section{Discussion}

The International SCI Pediatric A\&P Basic Data Set records the minimum amount of clinically relevant information on activities and participation of children with SCI between 6 and 17 years of age. The data set can be administered in conjunction with the newly developed International SCI Pediatric Education Data Set [26] to record the most relevant areas of function and participation in children with SCI.

None of the International SCI Basic Data Sets, including the International SCI Pediatric A\&P Basic Data Set were developed as assessments or outcome instruments. However, because they contain standardized methods for recording variables and are designed to facilitate pooling of clinical data, they should be validated and tested for reliability. Likewise, while the process by which the international SCI basic data sets are developed provides face validity, further evaluation for cultural relevance is necessary. Thus, future field testing of the International SCI Pediatric A\&P Basic Data Set is warranted, particularly for cultural relevance and feasibility of administration. If translated, it should be done so in accordance with the procedures outlined by BieringSørensen et al. [42]. As has been done for the other international SCI basic data sets, we anticipate that this data set will be exposed to a formal review sometime in the future after the field has had an opportunity to use it, and as our understanding and ability to measure activity and participation in children with SCI advance.

\section{Conclusion}

The International SCI Pediatric A\&P Basic Data Set provides an important addition to the library of international SCI basic data sets. It provides a mechanism to record activity and participation in a standardized fashion and therefore supports the pooling of clinical information from across the globe. The International Spinal Cord Injury Pediatric Activity and Participation Data Basic Data Set can be found on the ISCoS website (https://www.iscos.org.uk/ international-sci-data-sets).

Acknowledgements The working group greatly appreciates Kelly Arbour-Nicitopoulos for providing insight and recommendations for the physical activities domain of the International SCI Pediatric A\&P Basic Data Set, Andrea Berhman, Fin Biering-Sørensen, Michael DeVivo, Carol Haywood, Linda Jones, Bethany Lipa, Rebecca Martin, and Kathy Zebracki for the contributions in the development of the data set, and individuals who provided feedback in anonymity during the public review period.

\section{Compliance with ethical standards}

Conflict of interest SC receives research support from the Craig $\mathrm{H}$. Neilsen Foundation, the US Department of Defense Spinal Cord Injury 
Research Program and from the National Institute on Disability, Independent Living and Rehabilitation Research. MJM receives research support from the Shiners Hospitals for Children, National Institute Neurological Disease and Stroke, The Craig H. Neilsen Foundation and The Rick Hansen Institute. She receives compensation for her role as Associate Editor for Topics in Spinal Cord Rehabilitation, royalties for the text book "The Child and Young Adult with Spinal Cord Injury." LCV receives compensation for his role as editor for topics in spinal cord rehabilitation, royalties for the text book "The Child and Young Adult with Spinal Cord Injury." Dr Noonan is employed by Rick Hansen Institute, the funding source of this work. The remaining authors declare no conflict of interest.

Ethics approval All the authors affirm that the paper is an authentic, accurate, and transparent account of the study; no aspects of the study have been omitted; and the incongruities from the planned study have been explained. Ethical standards for this human subject's research were strictly followed.

Publisher's note Springer Nature remains neutral with regard to jurisdictional claims in published maps and institutional affiliations.

\section{References}

1. Biering-Sørensen F, Charlifue S, DeVivo M, Noonan V, Post M, Stripling T, et al. International spinal cord injury data sets. Spinal Cord. 2006;44:530-4.

2. International SCI Data Sets. http://www.iscos.org.uk/internationa 1-sci-data-sets. Retrieved 5 Jan 2019.

3. Biering-Sørensen F, Charlifue S, DeVivo MJ, Grinnon S, Kleitman N, Lu Y, et al. Using the spinal cord injury common data elements. Top Spinal Cord Inj Rehabil. 2012;18:23-7.

4. Biering-Sørensen F, Charlifue S, Devivo MJ, Grinnon ST, Kleitman N, Lu Y. et al. Incorporation of the International Spinal Cord Injury Data Set elements into the National Institute of Neurological Disorders and Stroke Common Data Elements. Spinal Cord. 2011;49:60-4.

5. Carroll A, Vogel LC, Zebracki K, Noonan VK, Biering-Sorensen F, Mulcahey MJ. Relevance of the international spinal cord injury basic data sets to youth: an inter-professional review with recommendations. Spinal Cord. 2017;55:875-81.

6. Hardin AP, Hackell JM, Committee on practice and ambulatory medicine. Age limits of pediatrics. Pediatrics. 2017;140:e20172151.

7. Sawyer SM, Azzopardi PS, Wickremarathne D, Patton G. The age of adolscence. Lancet Child Adolscent Health. 2018;2:P223-228.

8. Krogh K, Emmanuel A, Perrouin-Verbe B, Korsten MA, Mulcahey MJ, Biering-Sørensen F. International spinal cord bowel function basic data set (version 2.0). Spinal Cord. 2017;55:692-8.

9. Dvorak MF, Wing PC, Fehlings MG, Vaccaro AR, Itshayek E, Biering-Sørensen F, et al. International spinal cord injury column basic data set. Spinal Cord. 2012;50:817-21.

10. Biering-Sørensen F, Burns AS, Curt A, Harvey LA, Mulcahey MJ, Nance PW, et al. International spinal cord injury musculoskeletal data set. Spinal Cord. 2012;50:797-802.

11. Post MW, Charlifue S, Biering-Sorensen F, Catz A, Dijkers MP, Horsewell J, et al. Development of the international spinal cord injury activity and participation basic data set. Spinal Cord. 2016;54:530-4.

12. WHO. International Classification of Functioning, Disability and Health. Geneva: WHO; 2001

13. Chien CW, Rodger S, Copley J, Skorka K. Comparative content review of children's participation measures using the International Classification of Functioning, Disability and Health-Children and Youth. Arch Phys Med Rehabil. 2014;95:141-52.
14. Coster W, Law M, Bedell G, Khetani M, Cousins M, Teplicky R. Development of the participation and environment measure for children and youth: conceptual basis. Disabil Rehabil. 2011;34: 238-46.

15. King GA, Law M, King S, Hurley P, Hanna S, Kertoy M, et al. Measuring children's participation and leisure activities: construct validation of th CAPE and PAC. Child Care Health Dev. 2007;33:28-39.

16. Kowalski KC, Crocker PRE, Faulkner RA. Validation of the Physical Activity Questionnaire for Older Children. Pediatr Exerc Sci. 1997;9:174-86.

17. McConachi H, Colver AF, Forsyth RJ, Jarvis SN, Parkinson KN. Participation of disabled children: how should it be characterised and measured? Disabil Rehabil. 2006;28:1157-64.

18. Mulcahey MJ, Calhoun CL, Kelly EH, Vogel LC. The spinal cord independence measure (SCIM)-III self report for youth. Spinal Cord. 2016;54:204-12.

19. Noreau L, Lepage C, Boissiere L, Picard R, Fougeyrollas P, Mathieu J, et al. Measuring participation in children with disabilities using the Assessment of Life Habits. Dev Med Child Neurol. 2007;49:666-71.

20. Rosenberg L, Jarus T, Bart O. Development and initial validation of the Children Participation Questionnaire (CPQ). Disabil Rehabil. 2010;32:1633-44.

21. Washington LA, Wilson S, Engel JM, Jensen MP. Development and preliminary evaluation of a pediatric measure of community integration: the Pediatric Community Participation Questionnaire (PCPQ). Rehabil Psychol. 2007;52:241-5.

22. Young NL, William JI, Yoshida KK, Wright JG. Measurement properties of the activities scale for kids. J Clin Epidemiol. 2000;53:125-37.

23. Ziviani J, Desha L, Feeney R, Boyd R. Measures of participation outcomes and environmental considerations for children with acquired brain injury: a systematic review. Brain Impair. 2010;11:93-112.

24. Kowalski KC, Crocker RE, Donen RM. The Physical Activity Questionnaire for Older Children (PAQ-C) and Adolescents (PAQ-A) Manual. Saskatoon: College of Kinesiology, University of Saskatchewan; 2004.

25. Mulcahey MJ, Slavin MD, Ni P, Vogel LC, Thielen CC, Coster WJ, et al. The Pediatric Measure of Participation (PMoP) short forms. Spinal Cord. 2016;54:1183-7.

26. Carney J, Fisher R, Augutis M, Charlifue S, Biering-Sørensen F, Höfers W, et al. Development of the international spinal cord injury/dysfunction education basic data set. Spinal Cord Series and Cases. (in press).

27. Mulcahey MJ, Calhoun C, Riley A, Haley S. Children's reports of activity and participation after sustaining spinal cord injury: a cognitive interviewing study. Dev Neurorehabil. 2009;12: 191-200.

28. World Health Organization. International classification of functioning, disability and health: version of children and youth. Geneva: World Health Organization; 2007.

29. Garton AF, Pratt C. Leisure activities of adolescent school students: predictors of participation and interest. $\mathrm{J}$ Adolesc. 1991;14:305-21.

30. Pawelko KA, McGafas AH. Leisure wellbeing among adolescent groups: time, choices, and self-determination. Park Recreat. 1997;32:26-39.

31. Medrich EA, Marzke C. Young adolescents and discretionary time use: the nature of life outside school. US Dist Columbia. 1991;6:55.

32. Rimmer JH, Rowland JL, Yamaki K. Obesity and secondary conditions in adolescents wtih disabilities: addressing the needs of an underserved population. J Adolesc Health. 2007;41:224-9.

33. Booth ML. Assessment of physical activity: an international perspective. Res Q Exerc Sport. 2000;71:S114-20. 
34. de Groot S, Andriaansen JJ, Tepper M, Snoek GJ, van der Woude LH, Post MW. Metabolic syndrome in people with long-standing spinal cord injury: associations with physical activity and capacity. Appl Physiol Nutr Meta. 2016;41:1192-6.

35. January AM, Zebracki K, Chlan KM, Vogel LC. Mental health and risk of secondary medical complications in adults with pediatriconset spinal cord injury. Top Spinal Cord Inj Rehabil. 2014;20:1-12.

36. Itzkovich M, Gelernter I, Biering-Sorensen F, Weeks C, Laramee MT, Craven BC, et al. The Spinal Cord Independence Measure (SCIM) version III: reliability and validity in a multi-center international study. Disabil Rehabil. 2007;29:1926-33.

37. Fekete C, Eriks-Hoogand I, Baumberger M, Catz A, Itzkovich M, Lüthi $\mathrm{H}$, et al. Development and validation of the self-report version of the Spinal Cord Independence Measure (SCIM III). Spinal Cord. 2013;51:40-7.

38. Mulcahey MJ, DiGiovanni N, Calhoun C, Homko E, Riley A, Haley S. Children's and parents' perspectives of activity performance and participation following spinal cord injury. Am J Occup Ther. 2010;64:605-13.

39. Mulcahey MJ, Calhoun C, Tian F, Ni P, Vogel L, Haley S. Evaulation of newly developed item banks for child reported outcomes of participation following spinal cord injury. Spinal Cord. 2012;50:915-9.

40. Martin Ginis KA, Phang SH, Latimer AE, Arbour-Nicitopoulos KP. Reliability and validity tests of the leisure time physical activity questionnaire for people with spinal cord injury. Arch Phys Med Rehabil. 2012;93:677-82.

41. Booth ML, Owen N, Bauman AE, Gore CJ. Retest reliability of recall measures of leisure time physical activity in Australian adults. Int J Epidemiol. 1996;25:153-9.

42. Biering-Sørensen F, Alexander MS, Burns S, Charlifue S, DeVivo M, Dietz V, et al. Recommendations for translation and reliability testing if the International Spinal Cord Injury Data Sets. Spinal Cord. 2011;49:357-60. 